\title{
BMJ A cohort study on the rate of Open progression of diabetic chronic kidney disease in different ethnic groups
}

\author{
Omer Ali, ${ }^{1}$ Atif Mohiuddin, ${ }^{1}$ Rohini Mathur, ${ }^{2}$ Gavin Dreyer, ${ }^{1}$ Sally Hull, ${ }^{2}$ \\ Muhammad M Yaqoob ${ }^{1}$
}

To cite: Ali 0, Mohiuddin A, Mathur $\mathrm{R}$, et al. A cohort study on the rate of progression of diabetic chronic kidney disease in different ethnic groups. BMJ Open 2013;3:e001855. doi:10.1136/bmjopen-2012001855

- Prepublication history for this paper are available online. To view these files please visit the journal online (http://dx.doi.org/10.1136/ bmjopen-2012-001855).

$\mathrm{OA}$ and $\mathrm{AM}$ contributed equally.

Received 26 July 2012 Revised 5 February 2013 Accepted 6 February 2013

This final article is available for use under the terms of the Creative Commons Attribution Non-Commercial 2.0 Licence; see http://bmjopen.bmj.com

For numbered affiliations see end of article.

\section{Correspondence to}

Professor Magdi M Yaqoob; m.m.yaqoob@qmul.ac.uk

\section{ABSTRACT}

Objective: To compare the rate of progression of diabetic chronic kidney disease in different ethnic groups. Design: Prospective longitudinal observational study. Participants: All new patients attending a tertiary renal unit in east London with diabetic chronic kidney disease between 2000 and 2007 and followed up till 2009 were included. Patients presenting with acute end-stage kidney failure were excluded.

Main outcome measures: The primary outcome was annual decline in the estimated glomerular filtration rate (eGFR) in different ethnic groups. Secondary end points were the number of patients developing end-stage kidney failure and total mortality during the study period.

Results: 329 patients (age 60 \pm 11.9 years, 208 men) were studied comprising 149 south Asian, 105 White and 75 Black patients. Mean follow-up was $6.0 \pm 2.3,5.0$ \pm 2.7 and $5.6 \pm 2.4$ years for White, Black and south Asian patients, respectively. South Asian patients were younger and had a higher baseline eGFR, but both systolic and diastolic blood pressures were higher in Black patients $(p<0.05)$. Baseline proteinuria was highest for the south Asian group followed by the White and Black groups. Adjusted linear regression analysis showed that an annual decline in eGFR was not significantly different between the three groups. The numbers of patients developing end-stage kidney failure and total mortality were also not significantly different between the three groups. ACE or angiotensin receptor blockers use, and glycated haemoglobin were similar at baseline and throughout the study period.

Conclusions: We conclude that ethnicity is not an independent factor in the rate of progression renal failure in patients with diabetic chronic kidney disease.

\section{INTRODUCTION}

Diabetic chronic kidney disease (DCKD) is one of the leading causes of end-stage kidney failure (ESKF), accounting for $21 \%$ of all such cases in the UK. ${ }^{1}$ Suboptimal glycaemic and blood pressure (BP) control, development of albuminuria and family history are important risk factors for the development of CKD in diabetic patients.

\section{ARTICLE SUMMARY}

Article focus

- The aim of the study is to compare the rate of progression of diabetic chronic kidney disease (CKD) in different ethnic groups.

- To examine the number of patients developing end-stage kidney failure (ESKF) and total mortality by ethnicity.

Key messages

- This study demonstrates that there is no significant interaction between ethnicity and the rate of progression of CKD in patients with diabetes mellitus.

- The prevalence of renal replacement therapy, indicating ESKF, and mortality was also similar between the different ethnic groups.

Strengths and limitations of this study

- The strengths of this study include the large number of cases and the prolonged period of follow-up.

- Weaknesses include combining ethnic subgroups into three broad categories, which may mask differences in treatment or progression.

There is a higher prevalence of diabetes mellitus (DM) in Black and south Asian populations. The prevalence of diabetes among Black Caribbean and Indian men has been reported to be approximately $10 \%$ compared with $4.3 \%$ in Whites. ${ }^{2} \mathrm{~A}$ recent study was conducted in one of the most ethnically diverse cities in the UK where the incidence of renal replacement therapy (RRT) for south Asian and Black groups was reported to be, respectively, 1.88 and 2.16 times greater than for White patients. ${ }^{3}$ Therefore, it is not surprising that a higher proportion of patients on RRT comes from ethnic minority groups compared to the UK White population $(17.8 \% \text { vs } 11 \%)^{4}$

Mortality is also 3.5 times higher in south Asian and Black populations with diabetes compared to Whites in England and Wales. ${ }^{5}$ 
The higher incidence of ESKF in south Asian and Black populations may be related to higher incidence and poorer control of diabetes and hypertension. However, it may also be related to faster progression of DCKD in ethnic minority populations. There are conflicting reports on progression of DCKD in these population subgroups. Higher rates of kidney function decline have been reported in some studies while others have failed to show this trend. ${ }^{6-9}$

Most studies comparing progression of DCKD in ethnically diverse populations have either been small or had a short duration of follow-up. The renal clinic at the Royal London Hospital in east London serves an ethnically diverse population in a large urban centre. The aim of our study is first to determine whether the rate of kidney function decline differs by ethnic group after controlling for demographic characteristics and clinical parameters known to be associated with progression of CKD, and second, to examine the number of patients developing ESKF and total mortality by ethnicity.

\section{MATERIALS AND METHODS \\ Population}

The study was conducted at the Royal London Hospital, which serves as a tertiary referral centre for three primary care trusts in east London: Newham, Tower Hamlets, and City and Hackney, with a combined general practitioner (GP) registered population of 885625 at the end of 2011. The prevalence of CKD among patients with diabetes, based on a local study, is $18 \% .^{10}$

All adult patients above the age of 18 years with biopsy proven or clinical diagnosis of DCKD (where all secondary causes were excluded) attending our kidney outpatient clinic were included in the study. Any other diagnosis of $\mathrm{CKD}$, and those presenting acutely with ESKF were excluded from the study.

Ethnicity was self-assigned by the patient. Patients were grouped according to the ethnic categories of the 2001 census. For the purpose of this study, patients of Indian, Bangladeshi and Pakistani ethnicity were analysed together as the south Asian subgroup. Patients of African and Caribbean ethnicity were grouped together to form the Black subgroup, though we recognise that these groupings may conceal underlying heterogeneity. Participants were recruited to the study from 2000 until 2007 and followed up prospectively until 2009. Patients left the study if they moved away, died, were started on dialysis or transplanted. The follow-up for the remaining patients was censored at the end of 2009.

\section{Clinical data collection}

Data were captured electronically using an inhouse renal information technology programme (File maker pro) at every clinic visit. This included BP measured in the sitting position using cuffs appropriate for individual and all clinical events and changes in medication.
Ethical approval was sought, but not required, due to the observational nature of the study utilising routinely collected, anonymised patient data.

Baseline variables were collected at the first clinic visit and subsequently updated at every clinic visit. The study variables were defined as follows: ischaemic heart disease (IHD) included patients who had a documented history of angina, myocardial infarction or cardiac revascularisation. Diabetic retinopathy included patients who had been diagnosed with the condition by the diabetic ophthalmology clinic. Peripheral vascular disease (PVD) included patients with a history of intermittent claudication or documented vascular disease on an angiogram, and cerebrovascular disease (CVD) included patients with a history of transient or persistent vascular neurological deficit.

Estimated glomerular filtration rate (eGFR) was calculated based on the four-variable Modification of Diet in Renal Disease (MDRD) equation which includes a correction for Black ethnicity. Serum creatine was measured using a Roche Modular Platform automated analyser. Proteinuria was determined using the protein creatine ratio, PCR (Roche Modular Platform automated analyser). PCR was only calculated for patients who had positive proteins on a urine dipstick, and thus individuals with no PCR data were considered to have a value less than 15. This was based on a separate unit practice development observation on all dipstick negative urine samples in diabetic patients who when analysed had PCR $<15$ (personal communication). Glycated haemoglobin (HbAlc) was used to assess glycaemic control (BioRad Turbo 2 automated analyser). Data on systolic BP (SBP) and diastolic BP (DBP), HbA1c, proteinuria and eGFR were collected every 6 months.

\section{Data coding}

Data were cleaned, coded and analysed using Stata 10 . Baseline variables were created for: average eGFR, SBP, DBP, PCR and HbAlc value. PCR was considered both as a linear variable and a binary variable (presence or absence of proteinuria at baseline defined as PCR $>15$ ). Medications were coded a constant variable (ever present vs never present) for ACE inhibitors (ACEi), angiotensin receptor blockers (ARBs) or dual blockade.

\section{Descriptive analysis}

Bivariate statistics outlining the population breakdown by ethnicity were conducted. Analysis of variance (ANOVA) was used to examine ethnic differences in the means of continuous variables, while $\chi^{2}$ tests were used to examine differences in ethnic differences for categorical variables.

\section{Statistical methods}

Unadjusted and adjusted linear regression models were used to determine whether the annual change in eGFR differed by ethnic group. For this analysis, the eGFR 
values collected every 6 months were collapsed together to form annual averages.

To investigate whether the annual decline in eGFR was modified by ethnicity, an interaction term between years of follow-up and ethnic group was utilised. The addition of an interaction term between ethnicity and 'year squared' to the linear model already containing an interaction between ethnicity and year was shown to be non-significant, thus suggesting to us to proceed with a model examining linear annual change in eGFR.

The adjusted analysis controlled for age at baseline, gender, presence or absence of vascular disease (defined as any prior diagnosis of CVD, PVD or IHD), presence or absence of drug treatment (defined as any prior prescription of ACEi, ARBs or dual blockade), presence or absence of proteinuria at baseline (defined as a baseline PCR value greater than 15) and baseline eGFR. In addition, the analysis included interaction terms to examine the effect of BP and HbAlc being controlled to target in each year of follow-up. The BP target was set at $\leq 130 / 80 \mathrm{~mm} \mathrm{Hg}$ and the HbAlc target was $\leq 7.5 \%$.

ANOVA was used to examine ethnic differences in the distribution of continuous variables, while $\chi^{2}$ tests were used for categorical variables. Finally, survival analysis using Cox regression was used to estimate ethnic differences in the risk of death adjusting for age and gender.

\section{RESULTS}

\section{Population}

Of a total of 356 eligible patients, a total of 329 patients with more than 6 years follow-up were included. Twenty-seven patients were excluded because 10 presented with ESKF and 17 patients had no ethnicity data. Less than $10 \%$ of the patients had missing data, which was attributed to missing clinics, and less than $5 \%$ were lost to follow-up due to death or relocation. The baseline characteristics are shown in table 1.

The south Asian group was the youngest, with a shorter duration of diabetes and lower baseline BP in comparison to Whites. The Black group has the shortest duration of diabetes but higher baseline SBP and DBP. Baseline eGFR was highest for the south Asian group and lowest for the White group. Mean HbAlc did not differ between ethnic groups, while baseline proteinuria was highest for the south Asian group followed by the White and Black groups. Prevalence of IHD was highest

Table 1 Baseline characteristics of study participants by ethnic group

\begin{tabular}{lcccr}
\hline Characteristic & White & Black & South Asian & p Value \\
\hline $\mathrm{N}$ & 105 & 75 & 149 & \\
Baseline measures & & & & \\
Mean age (years) & $61.3 \pm 12.6$ & $62.4 \pm 11.3$ & $57.8 \pm 11.4$ & 0.005 \\
Male (\%) & 61.5 & 66.2 & 65.8 & 0.741 \\
T2DM (\%) & 85 & 100 & 98 & 0.001 \\
Duration of diabetes (years) & $18.3 \pm 11.6$ & $13.4 \pm 7.1$ & $14.0 \pm 12.9$ & 0.013 \\
Duration of follow-up (years) & $6.0 \pm 2.3$ & $5.0 \pm 2.7$ & $5.6 \pm 2.4$ & 0.05 \\
Diabetic retinopathy (\%) & 84 & 81 & 76 & 0.110 \\
Mean eGFR (ml/min/1.73 ${ }^{2}$ ) & $38.1 \pm 19$ & $39.0 \pm 21$ & $44.3 \pm 21$ & 0.039 \\
Mean systolic BP (mm Hg) & $146.6 \pm 26$ & $158.3 \pm 23$ & $136.6 \pm 24$ & $<0.001$ \\
Systolic BP >130 mm Hg (\%) & 78.1 & 86.7 & 61.7 & 0.001 \\
Mean diastolic BP (mm Hg) & $76 \pm 11$ & $83 \pm 15$ & $75 \pm 11$ & 0.002 \\
Diastolic BP >80 (\%) & 46.7 & 53.3 & 37.6 & 0.445 \\
Mean HbA1c (\%) & $8.5 \pm 1.8$ & $8.8 \pm 2.2$ & $8.7 \pm 1.9$ & 0.833 \\
HbA1c>7.5\% (\%) & 67.6 & 69.3 & 71.1 & $<0.001$ \\
PCR>15 mg/mmol (\%) & 58.2 & 47.5 & 64.4 & 0.020 \\
Constant measures & & & 38.9 & 0.626 \\
IHD (\%) & 28.6 & 21.3 & 12.1 & 0.799 \\
PVD (\%) & 15.2 & 10.7 & 10.7 & 0.742 \\
CVD (\%) & 10.5 & 8.0 & 80.4 & 0.894 \\
Statin use (\%) & 83.8 & 80.0 & 73.7 & 0.519 \\
ACE use (\%) & 72.4 & 70.7 & 41.2 & 0.483 \\
ARB use (\%) & 34.3 & 40.0 & 20.1 & 0.609 \\
Dual blockade (\%) & 14.3 & 17.3 & 13.1 & 0.272 \\
Current smoker (\%) & 18.6 & & 38.9 & 0.612 \\
Outcome measures & & & 8.1 & \\
End-stage kidney failure (\%) & 32.4 & 44.0 & & \\
Death (\%) & 10.5 & 12.0 & & \\
\hline
\end{tabular}

${ }^{*}$ Mean values \pm SD.

$\mathrm{ARB}$, angiotensin receptor blocker; BP, blood pressure; CVD, cerebrovascular disease; eGFR, estimated glomerular filtration rate; HbA1c, glycated haemoglobin; IHD, ischaemic heart disease; PCR, protein creatine ratio; PVD, peripheral vascular disease; T2DM, type 2 diabetes mellitus. 
Table 2 Unadjusted regression for annual change in estimated glomerular filtration rate $(n=329)$

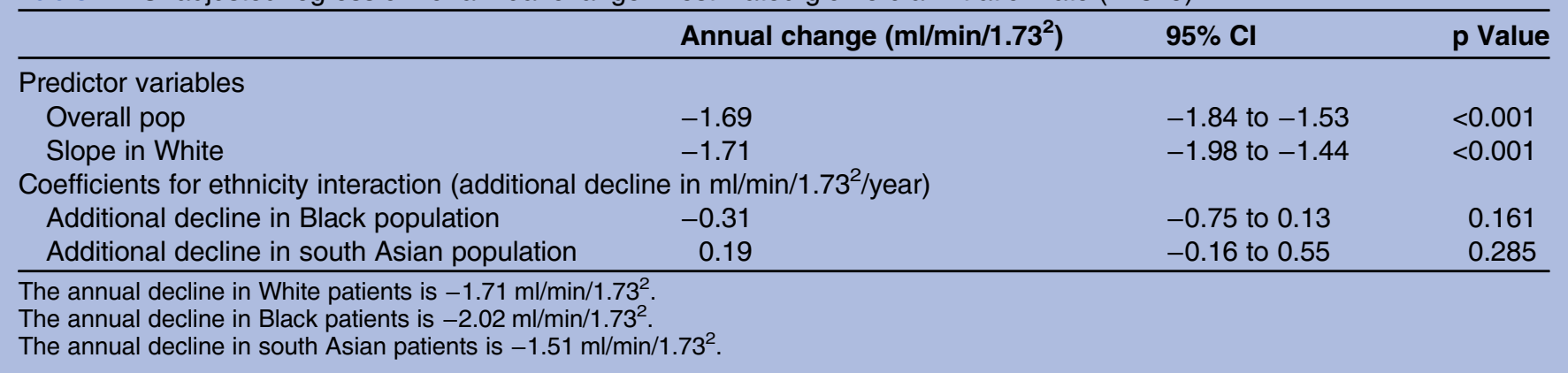

for the south Asian patients. Prevalence of other comorbidities, smoking and drug prescription was not significantly different between the three groups.

\section{Primary end point (annual decline in eGFR)}

The overall annual decrease in eGFR was -1.69 in the total population. In the unadjusted linear regression analysis, the annual decline in eGFR in the White, Black and south Asian groups was $-1.78,-2.02$ and -1.51 , respectively. There was no significant difference in the rate of decline in eGFR between the three groups (table 2).

Furthermore, the adjusted linear regression analysis indicated no significant differences in the annual change in eGFR between the ethnic groups over the entire study period. However, the analysis showed that control of BP, gender and degree of renal impairment at baseline were significant predictors of progression (table 3).

\section{Secondary end points}

By the end of the observation period, 125 individuals had developed ESKF, 32 individuals had died and 172 individuals were censored. The crude proportions of ESKF were $32 \%, 44 \%$ and $38 \%$ in the White, Black and south Asian groups, respectively, and were not statistically different $(\mathrm{p}=0.272)$. After adjusting for age and gender, the survival analysis indicated no significant difference by ethnicity in the risk of death (table 4 ).

\section{DISCUSSION}

In this single centre longitudinal prospective observational study of patients with DCKD, the average annual

Table 3 Adjusted linear regression for annual change in eGFR $(n=329)$

\begin{tabular}{|c|c|c|c|}
\hline & Annual change $\left(\mathrm{ml} / \mathrm{min} / 1.73^{2}\right)$ & $95 \% \mathrm{Cl}$ & p Value \\
\hline \multicolumn{4}{|l|}{ Predictor variables } \\
\hline Years of follow-up (slope in reference pop) & -1.93 & -2.31 to -1.56 & $<0.001$ \\
\hline \multicolumn{4}{|c|}{ Coefficients for ethnicity interaction (additional decline in $\mathrm{ml} / \mathrm{min} / 1.73^{2}$ per year) } \\
\hline Additional decline in Black population & -0.19 & -0.68 to 0.30 & 0.438 \\
\hline Additional decline in south Asian population & 0.08 & -0.31 to 0.48 & 0.676 \\
\hline \multicolumn{4}{|l|}{ Time-varying variables } \\
\hline $\mathrm{BP} \leq 130 / 80 \mathrm{~mm} \mathrm{Hg} \times$ time in years & 0.62 & 0.27 to 0.98 & $<0.001$ \\
\hline $\mathrm{HbA} 1 \mathrm{c} \leq 7.5 \% \times$ time in years & 0.03 & -0.33 to 0.39 & 0.883 \\
\hline \multicolumn{4}{|l|}{ Constant variables } \\
\hline \multicolumn{4}{|l|}{ Ethnicity (White is reference category) } \\
\hline Black ethnicity & -1.67 & -4.97 to 1.64 & 0.322 \\
\hline South Asian ethnicity & -0.51 & -3.25 to 2.23 & 0.716 \\
\hline Age & 0.03 & -0.06 to 0.13 & 0.466 \\
\hline Gender (female is reference category) & -2.64 & -4.87 to -0.40 & 0.021 \\
\hline SBP target at baseline & -2.31 & -3.89 to -0.73 & 0.004 \\
\hline $\mathrm{HbA} 1 \mathrm{c}$ value at baseline & -0.66 & -2.37 to 1.06 & 0.454 \\
\hline Vascular disease ever (PVD, CVD, IHD) & -1.44 & -3.66 to 0.79 & 0.206 \\
\hline Drug treatment ever (ACE, ARB, dual block) & 0.02 & -4.46 to 4.50 & 0.993 \\
\hline Proteinuria at baseline $(P C R>15)$ & 0.173 & -1.99 to 2.33 & 0.875 \\
\hline Baseline eGFR & 1.05 & 0.99 to 1.11 & $<0.001$ \\
\hline Constant & -3.18 & -11.26 to 4.89 & 0.440 \\
\hline
\end{tabular}

The annual decline in the reference population (White) is $-1.93 \mathrm{ml} / \mathrm{min}$.

The annual decline in Black patients is $-2.12 \mathrm{ml} / \mathrm{min}$.

The annual decline in south Asian patients is $-1.85 \mathrm{ml} / \mathrm{min}$.

ARB, angiotensin receptor blocker; BP, blood pressure; CVD, cerebrovascular disease; eGFR, estimated glomerular filtration rate; HbA1c, glycated haemoglobin; IHD, ischaemic heart disease; PCR, protein creatine ratio; PVD, peripheral vascular disease; SBP, systolic blood pressure 


$\begin{aligned} & \text { Table } 4 \text { Ethnic differences in risk of death rate using Cox } \\
& \text { regression }\end{aligned}$
\begin{tabular}{llll}
\hline Ethnic group & HR $^{*}$ & $\mathbf{9 5 \%} \mathbf{~ C l}$ & p Value \\
\hline White (ref) & 1 & - & - \\
Black & 1.01 & 0.63 to 2.57 & 0.982 \\
South Asian & 1.17 & 0.68 to 2.32 & 0.718 \\
\hline${ }^{*}$ Adjusted for age and gender. & &
\end{tabular}

decrease in eGFR in the whole population was $-1.69 \mathrm{ml} / \mathrm{min} / 1.73^{2}$. No significant differences in the rate of progression by ethnic group were evidenced. To date, this is the largest study of its kind in the literature.

Burden et $a l^{7}$ have reported previously that south Asians with diabetes are 13 times more likely to develop ESKF than their White counterparts, suggesting a faster progression of CKD. A study carried out in the UK, which supports this hypothesis, found that the proportion of patients doubling their creatine was significantly higher in south Asians compared to Whites. ${ }^{8}$ However, this study had significantly fewer patients than our study and their patients had early CKD at baseline compared with our population who had moderately severe renal impairment at presentation, which was an independent predictor of progression of renal failure. In contrast, another small study (39 patients) in the UK by Koppiker et $a .^{9}{ }^{9}$ has suggested no difference in the rate of progression between Whites and south Asians. ${ }^{9}$ However, complete data were available for only 36 patients. Similarly, a study in the USA has also reported no significant differences in progression of diabetic kidney disease between the Black and White populations with equivalent diabetic control. ${ }^{6}$ Our results are broadly in agreement with these studies by demonstrating a lack of interaction between ethnicity and the rate of decline in kidney function, though Black patients had a modest additional unadjusted annual decline of $0.31 \mathrm{ml} / \mathrm{min} / 1.73^{2}$.

$\mathrm{BP}$ and glycaemic control are important risk factors for progression of DCKD. Generally BP control is more difficult to achieve among Black populations and may contribute towards a faster rate of kidney function decline. One study showed that there was no significant difference in the rate of kidney function decline after adjusting for the effects of glycaemic control in Black populations compared with Whites, while others have shown significant differences despite equivalent $\mathrm{BP}$ and glycaemic control. A difference in the use of antihypertensive medication including renin angiotensin blocking medication (RAAS) has also been suggested. ${ }^{9} 1112$ In this study, there was no significant difference in glycaemic control or the use of RAAS in the three groups. However, Black patients had a significantly higher baseline SBP, DBP and above target BP. In our previous study, we observed a higher prevalence of $\mathrm{BP}>150 / 90$ in Blacks in a community-based cohort with mild CKD,${ }^{10}$ a result which is confirmed by this study in a hospital-based cohort of patients with DCKD. Although, there was no significant difference in the decline of kidney function between ethnic groups in this study, the adjusted analysis showed that the lack of control of BP below the target of $130 / 80 \mathrm{~mm} \mathrm{Hg}$ was independently associated with a rapid decline of eGFR underscoring the importance of optimal BP management as a key modifiable risk factor. Moreover, as expected, gender and severity of renal impairment were the other significant predictors of progression of diabetic kidney disease (table 3).

There was no significant difference in total mortality (table 4) and prevalence of ESKF between the different ethnic groups; however, the study was not powered to address this question adequately and will require a large cohort study to confirm these findings. It is important to note, however, that south Asians had a higher baseline eGFR, and this could be explained, in part, by ethnic differences in referral patterns to our centre. Primary care physicians may be more likely to refer south Asians with early diabetic kidney disease compared to Whites, reflecting concern that diabetic kidney disease in south Asians is more difficult to control and is associated with a faster rate of decline.

Current national CKD guidelines do not include ethnicity as a risk factor for CKD. ${ }^{13}$ Therefore, the recommendation for the management of CKD in general and DCKD in particular for patients from different ethnic minority groups is similar to that for the White population. The results of this study suggest that there is no need for a different set of guidelines for different ethnic groups at present, and efforts should be directed at determining the reasons behind suboptimal BP targets in certain ethnic groups.

\section{Strengths and weaknesses of the study}

The strengths of this study include the large number of cases, the completeness of ethnicity and clinical data recording and the prolonged period of follow-up. The communities from which these patients are drawn include some of the most socially deprived and multiethnic populations in England; hence, our results will be of interest to commissioning organisations throughout the UK.

Weaknesses include combining ethnic subgroups into three broad categories, which may mask differences in treatment or progression. We are also unable to identify differences in referral thresholds and patterns by the GP practices in the localities. Another perceived weakness of the study could be combining diabetic patients with types 1 and 2 for analysis. However, in a real-life setting, only differences in patients with CKD with types 1 and 2 are stages at which patients are referred because of the variable period of undiagnosed type 2 DM. However, regardless of the type of diabetes and the stage of renal involvement, achievement of SBP/DBP values of 130/ $80 \mathrm{~mm} \mathrm{Hg}$ or less and of HbAlc levels of $7.5 \%$ or less are of paramount importance for the beneficial effect which optimal BP and metabolic control may have on all the other macrovascular and microvascular chronic complications of diabetes. ${ }^{14}$ 


\section{CONCLUSION}

This study demonstrates that there is no significant interaction between ethnicity and the rate of progression of CKD in patients with DM. Furthermore, the prevalence of RRT, indicating ESKF, and mortality was also similar between the three groups.

\section{Author affiliations}

${ }^{1}$ Department of Nephrology, Royal London Hospital, London, UK ${ }^{2}$ Department of Primary Care and Public Health, Barts and The London School of Medicine and Dentistry, Queen Mary University of London, London, UK

Contributors All authors listed have contributed sufficiently to the project to be included as authors

Funding This research received no specific grant from any funding agency in the public, commercial or not-for-profit sectors.

Competing interests None.

Ethics approval This was an observational study, and the data were completely anonymised. Ethical committee chairman Dr Arthur Tucker advised us that it did not require ethical approval because it was collection of longitudinal data and patients were followed and treated as per standard guidelines.

Provenance and peer review Not commissioned; externally peer reviewed.

\section{REFERENCES}

1. Byrne C, Ford D, Gilg J, et al. UK ESRD Incident Rates in 2008: national and centre-specific analyses. UK Renal registry report 2009 http://www.renalreg.com/Reports/2009.html (accessed Feb 2013).
2. Health Survey of England. 2004. http://www.ic.nhs.uk/pubs/ hse04ethnic (accessed Feb 2013).

3. Lambie M, Richards N, Smith S. Ethnicity, age and incidence rates for renal replacement therapy in Birmingham, UK: 1990-2004. Nephrol Dial Transplant 2008;23:3983-7.

4. Ansell DFJ, Feest TG, UK Renal Registry Report. 2007. http://www. renalreg.com (accessed Feb 2013).

5. Raleigh VS, Kiri V, Balarajan R. Variations in mortality from diabetes mellitus, hypertension and renal disease in England and Wales by country of birth. Health Trends 1997;28:122-7.

6. Salifu M, Shah S, lqbal M, et al. Effect of ethnicity on the progression of diabetic kidney disease independent of glycemic control. Am J Nephrol 2009;30:261-7.

7. Burden AC, McNally PG, Feehally J, et al. Increased incidence of end-stage renal failure secondary to diabetes mellitus in Asian ethnic groups in the United Kingdom. Diabet Med 1992:9:641-5.

8. Earle KK, Porter KA, Ostberg J, et al. Variation in the progression of diabetic nephropathy according to racial origin. Nephrol Dial Transplant 2001:16:286-90.

9. Koppiker N, Feehally J, Raymond N, et al. Rate of decline in renal function in Indo-Asians and Whites with diabetic nephropathy. Diabet Med 1998;15:60-5.

10. Dreyer G, Hull S, Aitken Z, et al. The effect of ethnicity on the prevalence of diabetes and associated chronic kidney disease. QJM 2009;102:261-9.

11. Duru O, Li S, Jurkovitz C, et al. Race and sex differences in hypertension control in CKD: results from the kidney early evaluation program (KEEP). Am J Kidney Dis 2008;51:192-8.

12. Davis TM. Ethnic diversity in type 2 diabetes. Diabet Med 2008;25 (Suppl 2):52-6.

13. Joint Specialty Committee on Renal Medicine of the Royal College of Physicians and the Renal Association and the Royal College of General Practitioners. Chronic Kidney Disease in Adults: UK Guidelines for Identification, Management and Referral. London: Royal College of Physicians, 2006.

14. Ritz E, Orth SR. Nephropathy in patients with type 2 diabetes mellitus. N Engl J Med 1999;341:1127-33. 